\title{
Duração do período de molhamento foliar: Medição e estimativa em feijão sob diferentes tratamentos hídricos
}

\author{
Angelica Durigon' \& Quirijn de J. van Lier ${ }^{2}$
}

\begin{abstract}
RESUMO
A variável duração do período de molhamento foliar desempenha papel importante em sistemas de alerta fitossanitários e em estudos de trocas gasosas das plantas. Objetivou-se, neste estudo, estimar essa variável por modelos em um dossel de feijão (Phaseolus vulgaris L.) sob irrigação plena e deficitária e comparar tais estimativas com medidas feitas no dossel. Foram utilizados quatro modelos: (1) o do número de horas com umidade relativa superior a um limiar; (2) o da depressão do ponto de orvalho; (3) o da árvore de classificação e regressão e (4) o modelo físico de Penman-Monteith. Os dois primeiros modelos apresentaram os menores erros quando os limiares empíricos foram iguais a 83-84\% e 2,6-2,7 ${ }^{\circ} \mathrm{C}$; os erros para o terceiro modelo foram da mesma ordem de grandeza dos obtidos pelos demais modelos uma vez que o mesmo utiliza limiares empíricos de três variáveis. Os erros para o modelo de Penman-Monteith foram reduzidos quando as entradas foram a temperatura e a umidade relativa do ar medidas no tratamento sob irrigação deficitária. Para as simulações com os dados do tratamento com irrigação plena os erros deste modelo se mantiveram próximos aos obtidos para as simulações feitas com os dados medidos da estação meteorológica.
\end{abstract}

Palavras-chave: orvalho, modelos empíricos, modelo físico

\section{Leaf wetness duration: Measurements and estimation in a bean crop under different water treatments}

\begin{abstract}
Leaf wetness duration variable plays an important role in crop protection warning systems and in studies of gas exchange in plants. The main objective of this study was to estimate the leaf wetness duration in a bean crop (Phaseolus vulgaris L.) by modeling under full and deficit irrigation and to compare it with field measurements. Four models were tested: (1) the model of the number of hours with relative humidity above a threshold; (2) the model of dew point depression; (3) the model of classification and regression tree, and (4) the model of Penman-Monteith. The first two models presented the lowest statistical errors when the empirical thresholds were equal to $83-84 \%$ and $2.6-2.7{ }^{\circ} \mathrm{C}$. The statistical errors of the third model were nearly the same as those obtained for the other models since it uses three empirical thresholds. The errors for the Penman-Monteith model were reduced when the air temperature and humidity input data were measured in the deficit irrigation treatment. For the Penman-Monteith model simulations with the fully irrigated treatment data, the errors remained close to those observed for the simulations with the weather station data.
\end{abstract}

Key words: dew, empirical models, physical model 


\section{INTRODUÇÃO}

No ambiente natural a superfície das folhas das plantas é frequentemente coberta por água na forma líquida. $\mathrm{O}$ termo "molhamento foliar" abrange todas as formas de água líquida na superfície das folhas das plantas, com origem na formação de orvalho, na precipitação ou na irrigação. Do molhamento foliar derivou-se a variável agrometeorológica Duração do Período de Molhamento foliar (DPM), parâmetro importante em sistemas de alerta fitossanitários (Kim et al., 2006; Lulu, 2008; Marcuzzo et al., 2009) e em estudos de redução das trocas gasosas da fotossíntese e da transpiração (Ishibashi \& Terashima, 1995; Hanba et al., 2004; Letts \& Mulligan, 2005).

A DPM provocada pela deposição de orvalho é uma variável de difícil medida ou estimativa em virtude de não estar relacionada somente às variáveis meteorológicas mas também ao microclima e às propriedades ópticas da superfície na qual ocorre o molhamento. Não há um padrão para a observação da DPM, tanto em termos de sensores como da sua própria instalação, inviabilizando estudos comparativos dessa variável entre locais de diferentes climas, por exemplo (Sentelhas, 2004). Os sensores mais atuais para a medida do molhamento foliar são eletrônicos, nos quais a formação de água líquida sobre uma placa promove a mudança na resistência ou impedância elétrica do elemento sensor.

A estimativa da DPM por modelos empíricos (Gleason et al., 1994; Kim et al., 2005) ou físicos (Sentelhas et al., 2006; Sentelhas \& Gillespie, 2008; Bregaglio et al., 2011) é, por outro lado, uma alternativa viável embora em muitos casos sejam necessárias, para seus cálculos, variáveis meteorológicas que não são normalmente medidas, como é o caso do saldo de radiação ou, ainda, carecerem de ajustes e calibrações para diferentes climas locais e culturas (Weiss et al., 1989; Huber \& Itier, 1990; Papastamati et al., 2004).

Dos modelos empíricos de estimativa da DPM, destaca-se o modelo do número de horas com umidade relativa acima de determinado limiar (NHUR) (Sentelhas, 2004; Lulu, 2008). Geralmente se adota este limiar como sendo $90 \%$, embora possa variar com condição meteorológica do local. O modelo empírico da depressão da temperatura do ponto de orvalho (DPO) dado pela diferença entre a temperatura do ar e a temperatura do ponto de orvalho, estima a DPM pelo período em que a DPO permanece abaixo do limiar de $2{ }^{\circ} \mathrm{C}$, tradicionalmente adotado mas que também pode variar de acordo com a condição meteorológica do local. O modelo empírico CART (modelo de árvore de classificação e regressão) sugerido por Gleason et al. (1994), estima a DPM a partir de uma combinação de limiares da DPO, da umidade relativa do ar e da intensidade do vento a $10 \mathrm{~m}$ através de uma árvore de classificação binária que apresenta categorias e ramos para distinguir se um intervalo de tempo apresenta ou não molhamento.

O modelo físico de Penman-Monteith (Sentelhas et al., 2006) ou de resistência aerodinâmica, tem aplicação universal, ao contrário dos modelos empíricos. Este modelo presume que a temperatura do ar medida entre 1,5 e 2 m sobre o gramado em uma estação meteorológica, representa a temperatura do ar na mesma altura sobre uma cultura. Para tanto, uma resistência aerodinâmica, função da altura da cultura e descrita por Monteith \& Unsworth (1990) é adicionada ao modelo.
Objetivou-se, no estudo, estimar a DPM em um dossel de feijão (Phaseolus vulgaris L.) sob duas condições hídricas utilizando-se quatro modelos (três empíricos e um físico) e comparar tais estimativas com medidas da DPM feitas no dossel. O efeito da condição meteorológica local em cada tratamento de irrigação na formação do orvalho também foi avaliado.

\section{Material e Métodos}

A área experimental utilizada para o experimento de campo localiza-se no Campus da Escola Superior de Agricultura "Luiz

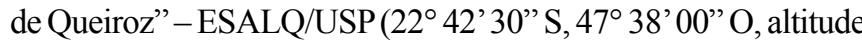
de $546 \mathrm{~m}$ ) cujo solo é do tipo Nitossolo vermelho. A semeadura de feijão, cultivar Pérola, foi feita em 11 de junho/2010 e a colheita realizada em 23 de setembro/2010. A área, de aproximadamente $990 \mathrm{~m}^{2}(22 \times 45 \mathrm{~m})$, foi dividida em duas parcelas $(22 \times 22,5 \mathrm{~m})$ em que uma foi irrigada durante todo ciclo da cultura (tratamento de irrigação plena - IP) enquanto a outra foi mantida sob déficit hídrico a partir da fase reprodutiva (tratamento de irrigação deficitária - ID); para tanto, um sistema de irrigação por aspersão foi montado na área experimental.

A estação meteorológica automatizada da ESALQ/USP está localizada a $20 \mathrm{~m}$ da área experimental e forneceu os dados de temperatura $\left(\mathrm{T}_{\mathrm{ar}},{ }^{\circ} \mathrm{C}\right)$ e umidade relativa do ar (UR, \%), precipitação $(\mathrm{P}, \mathrm{mm})$, saldo de radiação $\left(\mathrm{R}_{\mathrm{n}}, \mathrm{W} \mathrm{m}^{-2}\right)$ e intensidade do vento a $2 \mathrm{~m}$ de altura $\left(\mathrm{U}, \mathrm{m} \mathrm{s}^{-1}\right)$ durante o experimento. As medidas de temperatura e umidade relativa do ar, embora disponíveis na estação meteorológica, foram também realizadas no centro de cada tratamento, a uma altura de $2 \mathrm{~m}$. Os dois sensores utilizados para essas medidas, da marca Campbell, modelo CS215-L9 ${ }^{\circledR}$, foram conectados a um armazenador de dados, modelo CR $1000^{\circledR}$, da mesma marca. As medidas de umidade relativa foram realizadas instantaneamente a cada 30 min enquanto as medidas de temperatura do ar são o resultado da média obtida durante o mesmo período. Os dados de temperatura e a umidade relativa do ar obtidos sobre o dossel foram utilizados como entrada nos modelos de estimativa da DPM.

A presença de água líquida proveniente da formação de orvalho nas folhas das plantas foi medida por dois Leaf Wetness Sensors da marca Decagon Devices, modelo LWS-L ${ }^{\circledR}$, instalados um em cada tratamento. Tais sensores foram conectados ao armazenador de dados CR $1000^{\circledR}$ e as medidas, registradas a cada $30 \mathrm{~min}$, foram dadas em períodos de minutos em que a placa, simulando a folha, estava seca ou molhada. Os LWS-L ${ }^{\circledR}$ foram instalados a $0,45 \mathrm{~m}$ do solo com ângulo de $0^{\circ} \mathrm{em}$ relação à superfície e mantidos nesta posição por todo o ciclo da cultura.

Durante os 104 dias do experimento de campo ocorreu precipitação nos dias 13,14 e 15 de julho $(62,9 \mathrm{~mm})$ em 7 de setembro $(12,8 \mathrm{~mm})$ e em 21 de setembro $(38,0 \mathrm{~mm})$ como mostrado na Figura 1. Durante mais de 50 dias, entre 16 de julho e 6 de setembro, não houve precipitação fazendo com que o solo atingisse condições secas. A aplicação de irrigação foi mantida nos dois tratamentos até o início do período reprodutivo e encerrada no dia 3 de setembro. Nos períodos entre 2 e 24 de agosto e entre 27 de agosto e 2 de setembro, somente o tratamento IP recebeu irrigação; no dia 24 de agosto 
foi realizada uma última irrigação no tratamento ID para garantir a sobrevivência das plantas; o período entre 3 de agosto e 2 de setembro foi definido como o período de estudo.

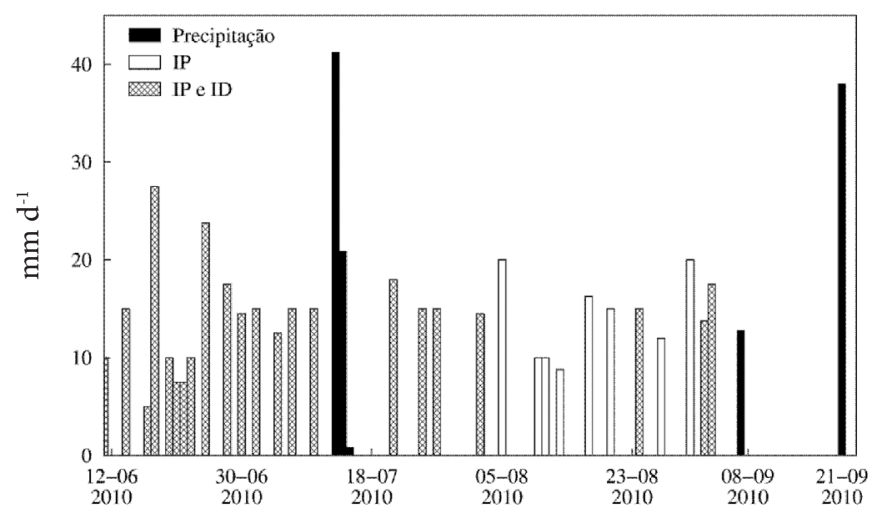

Figura 1. Ocorrência de precipitação e irrigações aplicadas nos tratamentos de irrigação plena (IP) e deficitária (ID) durante o experimento de campo

A altura média das plantas do tratamento IP durante o período de estudo foi igual a $0,6 \mathrm{~m}$ enquanto no tratamento ID foi igual a $0,5 \mathrm{~m}$. Esses valores foram obtidos através da média de três medidas feitas em 10 plantas por tratamento, nos dias 4 e 19 de agosto e no dia 2 de setembro.

Dentre os diferentes modelos de estimativa da DPM três modelos empíricos e um modelo físico foram selecionados para o presente estudo.

Dos modelos empíricos o modelo do número de horas com umidade relativa acima de determinado limiar (NHUR) se destaca pela aplicabilidade uma vez que utiliza o limiar de uma única variável meteorológica (umidade relativa do ar) para estimar a DPM. O número de horas com a umidade relativa do ar acima de 90\% (NHUR > 90\%) é tradicionalmente adotado embora a calibração desse limiar empírico para o clima do local no qual a DPM está sendo estimada e para a cultura na qual ocorre o molhamento seja recomendada (Sentelhas, 2004). A soma dos minutos com umidade relativa do ar acima de $90 \%$ dividida por 60 foi considerada como a DPM expressa em horas.

O modelo empírico da depressão da temperatura do ponto de orvalho (DPO) foi sugerido por Gillespie et al. (1993). Tratase do número de horas em que a diferença entre a temperatura do ar e a temperatura do ponto de orvalho, conhecida como a depressão da temperatura do ponto de orvalho, permaneceu entre dois limiares específicos, tradicionalmente adotados como sendo $2{ }^{\circ} \mathrm{C}$ para a deposição do orvalho e $3,8{ }^{\circ} \mathrm{C}$ para sua secagem; para o modelo DPO também se recomenda que os limiares empíricos sejam calibrados localmente.

O modelo empírico de árvore de classificação e regressão (CART) foi desenvolvido para estimar a DPM a partir da DPO, da intensidade do vento a $10 \mathrm{~m}$ e da umidade relativa do ar (Gleason et al., 1994). A árvore de classificação binária utilizada pelo modelo CART, que apresenta categorias e ramos para determinar se uma combinação das variáveis de entrada com determinados limiares é capaz de formar o orvalho, é apresentada detalhadamente em Sentelhas (2004). Para a utilização do modelo CART o vento medido na altura de $2 \mathrm{~m}$ na estação meteorológica foi convertido para a altura de $10 \mathrm{~m}$ através da relação logarítmica, descrita em Allen et al. (1998).

Além dos três modelos empíricos acima descritos o modelo físico de Penman-Monteith também foi utilizado neste estudo. O fluxo de calor latente ( $\mathrm{LE}, \mathrm{kJ} \mathrm{m}^{-2} \mathrm{~s}^{-1}$ ) para uma folha artificial pode ser estimado, portanto, para cada intervalo de tempo (30 min, neste caso) pela equação de Penman-Monteith:

$$
L E=-\frac{\Delta R_{n}+\left[\frac{\rho_{a} c_{p}\left(e_{s}-e_{a}\right)}{r_{a}+r_{b}}\right]}{\Delta+\gamma}
$$

em que: $\Delta$ é a tangente à curva de pressão de vapor do ar saturado $\left(\mathrm{kPa} \mathrm{K}^{-1}\right)$ no ponto correspondente à temperatura do ar, $\mathrm{R}_{\mathrm{n}}\left(\mathrm{kJ} \mathrm{m}^{-2} \mathrm{~s}^{-1}\right)$ é a radiação líquida, $\rho_{\mathrm{a}}$ é a densidade do ar seco $\left(\mathrm{kg} \mathrm{m}^{-3}\right), \mathrm{c}_{\mathrm{p}}$ é o calor específico isobárico do $\operatorname{ar}\left(\mathrm{kJ} \mathrm{kg}^{-1} \mathrm{~K}^{-1}\right), \mathrm{e}_{\mathrm{s}}$ $(\mathrm{kPa})$ e $\mathrm{e}_{\mathrm{a}}(\mathrm{kPa})$ são, respectivamente, a pressão de saturação na temperatura do ar da estação e real do vapor d'água, $\gamma$ é a constante psicrométrica, assumida constante e igual a $0,64 \mathrm{kPa} \mathrm{K}^{-1}$ para períodos com orvalho, $\mathrm{r}_{\mathrm{a}}\left(\mathrm{s} \mathrm{m}^{-1}\right)$ é a resistência aerodinâmica, e $r_{b}\left(\mathrm{~s} \mathrm{~m}^{-1}\right)$ é a resistência da cultura para o transporte de calor. Para a estimativa de $\mathrm{r}_{\mathrm{b}}$, é necessário conhecer a dimensão efetiva da folha artificial adotando-se o valor igual a $0,06 \mathrm{~m}$. Por este modelo a DPM é dada pelo intervalo de tempo em que LE $>0$ e, visto que não ocorreu precipitação durante o período de estudo e os horários de irrigação foram excluídos da análise, todos os intervalos de tempo com LE > 0 são decorrentes da formação de orvalho. $\mathrm{O}$ coeficiente de molhamento geral $\mathrm{W}_{\mathrm{g}}$, descrito em Lulu (2008), foi utilizado para converter a DPM estimada sobre o gramado pelo modelo de Penman-Monteith, em DPM na cultura. As estimativas da DPM com o modelo de Penman-Monteith também foram feitas com as medidas de temperatura e umidade relativa do ar sobre o dossel, em cada um dos tratamentos hídricos. Os erros nas estimativas da DPM com os dados da estação meteorológica e com os dados obtidos sobre o dossel foram analisados.

A DPM foi computada considerando-se o número de intervalos de $30 \mathrm{~min}$ entre as $12 \mathrm{~h}$ e $30 \mathrm{~min}$ do dia $\mathrm{n}$ e às 12 $\mathrm{h}$ do dia $\mathrm{n}+1$ nos quais os modelos estimaram a presença de orvalho. Os períodos diários se iniciaram às $12 \mathrm{~h}$ e $30 \mathrm{~min}$, ao invés da meia-noite, para evitar a separação temporal de um mesmo evento (noturno) de formação de orvalho.

Para avaliar o desempenho das estimativas feitas pelos modelos em comparação às observações, calcularam-se os seguintes índices estatísticos:

1. Erro médio (EM, h):

$$
\mathrm{EM}=\sum_{\mathrm{i}=1}^{\mathrm{n}} \frac{\left(\mathrm{DPM}_{\mathrm{e}, \mathrm{i}}-\mathrm{DPM}_{\mathrm{o}, \mathrm{i}}\right)}{\mathrm{n}}
$$

2. Erro médio absoluto (EMA, h):

$$
\mathrm{EMA}=\sum_{\mathrm{i}=1}^{\mathrm{n}} \frac{\left(\left|\mathrm{DPM}_{\mathrm{e}, \mathrm{i}}-\mathrm{DPM}_{\mathrm{o}, \mathrm{i}}\right|\right)}{\mathrm{n}}
$$

3. Erro máximo absoluto (EMax, h): 


$$
\operatorname{EMax}=\max \left\{\left|\mathrm{DPM}_{\mathrm{e}, \mathrm{i}}-\mathrm{DPM}_{\mathrm{o}, \mathrm{i}}\right|\right\}_{\mathrm{i}=1}^{\mathrm{n}}
$$

4. Raiz quadrada do erro quadrático médio das previsões $\left(\mathrm{REQM}_{\mathrm{p}}, \mathrm{h}\right)$ :

$$
\operatorname{REQM}_{p}=\sqrt{\sum_{\mathrm{i}=1}^{\mathrm{n}} \frac{\left(\mathrm{DPM}_{\mathrm{e}, \mathrm{i}}-\mathrm{DPM}_{\mathrm{o}, \mathrm{i}}\right)^{2}}{\mathrm{n}}}
$$

5. Coeficiente de eficiência das previsões (E, -) (Nash \& Sutcliffe, 1970):

$$
E=1,0-\frac{\sum_{i=1}^{n}\left(\mathrm{DPM}_{e, i}-\mathrm{DPM}_{\mathrm{o}, \mathrm{i}}\right)^{2}}{\sum_{\mathrm{i}=1}^{\mathrm{n}}\left(\mathrm{DPM}_{\mathrm{o}, \mathrm{i}}-\overline{\mathrm{DPM}}_{\mathrm{o}}\right)^{2}}
$$

em que:

$\mathrm{DPM}_{\mathrm{o}, \mathrm{i}}$ e $\mathrm{DPM}_{\mathrm{e}, \mathrm{i}}$ - i-ésimo valor da Duração do Período de Molhamento foliar medida no campo e estimada por um modelo, respectivamente; e

$\overline{\mathrm{DPM}}_{\mathrm{o}}$ - média da DPM medida, ambas as variáveis dadas em horas.

$\mathrm{O}$ índice adimensional $\mathrm{E}$ fornece uma medida relativa do desempenho do modelo variando de $-\infty$ a 1 , sendo que valores próximos a 1 indicam que o modelo tem melhor desempenho na representação dos dados observados do que a média das próprias observações. Os índices EM, EMA, EMax e REQM, por sua vez, fornecem uma análise do erro na unidade de medida da variável analisada. A raiz quadrada do erro quadrático médio das observações (REQM, h) também foi calculada para fins de comparação:

$$
R E Q M=\sqrt{\sum_{i=1}^{n} \frac{\left(D_{0, i}-\overline{D P M}_{o}\right)^{2}}{n}}
$$

\section{Resultados e Discussão}

Apesar da proximidade entre os tratamentos o efeito microclimático da diferença da transpiração das plantas irrigadas e das plantas sob déficit hídrico pode ser observado na diferença entre as variáveis meteorológicas medidas em ambos os tratamentos. Na Figura 2 são apresentadas as diferenças de temperatura e umidade relativa do ar e a depressão do ponto de orvalho entre os tratamentos de irrigação plena (IP) e deficitária (ID). No início do período de estudo (início do mês de agosto) a temperatura do ar no tratamento ID era aproximadamente $0,3{ }^{\circ} \mathrm{C}$ superior à do tratamento IP, mesmo que esta diferença tenha aumentado até o final do mês, conforme o efeito do déficit hídrico nas plantas do tratamento ID tenha se acentuado. A temperatura média do ar, tanto nos dois tratamentos do dossel quanto na estação meteorológica, foi aproximadamente igual a $19{ }^{\circ} \mathrm{C}$ durante o período de estudo. A umidade relativa do ar no tratamento ID estava ligeiramente maior do que no tratamento IP $(\sim 1 \%)$ no início do período de estudo reduzindo no decorrer
A.

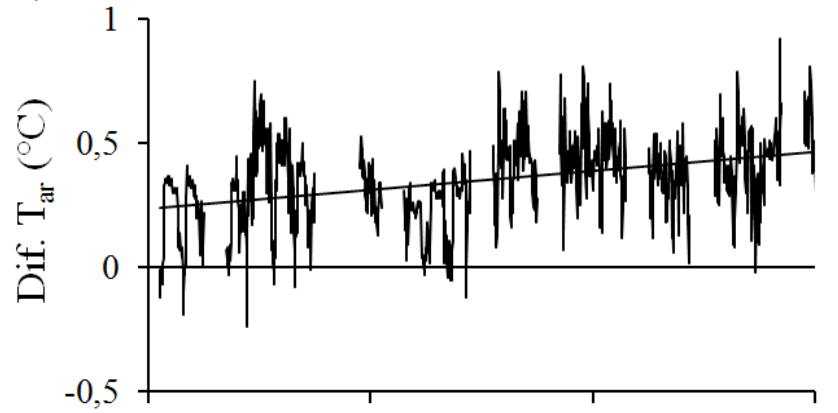

B

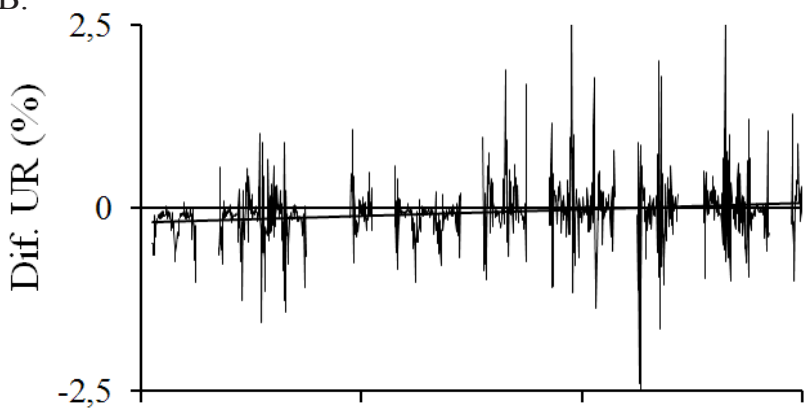

C.

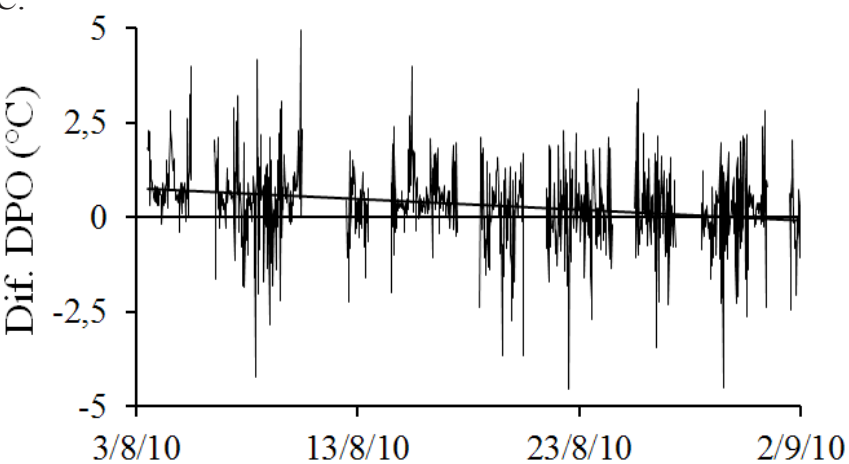

Figura 2. Diferença de temperatura do $\operatorname{ar} \mathrm{T}_{\mathrm{ar}}\left({ }^{\circ} \mathrm{C}\right)$, umidade relativa do ar UR (\%) e depressão da temperatura do ponto de orvalho DPO $\left({ }^{\circ} \mathrm{C}\right)$ entre os tratamentos de irrigação plena e deficitária (IP - ID)

do período inversamente à temperatura do ar, conforme mostra a linha de tendência. A umidade relativa do ar média foi igual a $59 \%$ em ambos os tratamentos durante o período e $5 \%$ mais elevada (64\%) nas medições feitas na estação meteorológica da ESALQ/USP. No início do mês de agosto, quando as condições hídricas nos dois tratamentos ainda eram semelhantes, a depressão da temperatura do ponto de orvalho foi levemente maior no tratamento IP do que no tratamento ID, ou seja, havia a maior chance de formação de orvalho no tratamento ID; entretanto, com o decorrer do mês e conforme o teor de água no solo do tratamento ID foi reduzido e a DPO tornou-se maior neste tratamento em comparação com o tratamento IP desfavorecendo, assim, a formação de orvalho.

Dentre as variáveis meteorológicas o vento é o principal responsável pelo secamento da água condensada nas folhas (Magareyet al., 2005). Por esta razão, além da temperatura e umidade relativa do ar e da depressão da temperatura do ponto de orvalho, o vento também é uma das variáveis de entrada nos modelos de estimativa da DPM. As medidas de vento feitas na estação meteorológica da ESALQ/USP utilizadas neste estudo 
são apresentadas na Figura 3. Na primeira metade do mês foram observadas rajadas de vento de até $4,9 \mathrm{~m} \mathrm{~s}^{-1} \mathrm{e}$ a partir do dia 19 de agosto, período em que não houve ocorrência de fenômenos meteorológicos mais intensos, a intensidade do vento permaneceu praticamente constante, com intensidade máxima de $2,1 \mathrm{~m} \mathrm{~s}^{-1}$ até o dia 31 de agosto, quando uma frente fria se aproximou da região de Piracicaba.

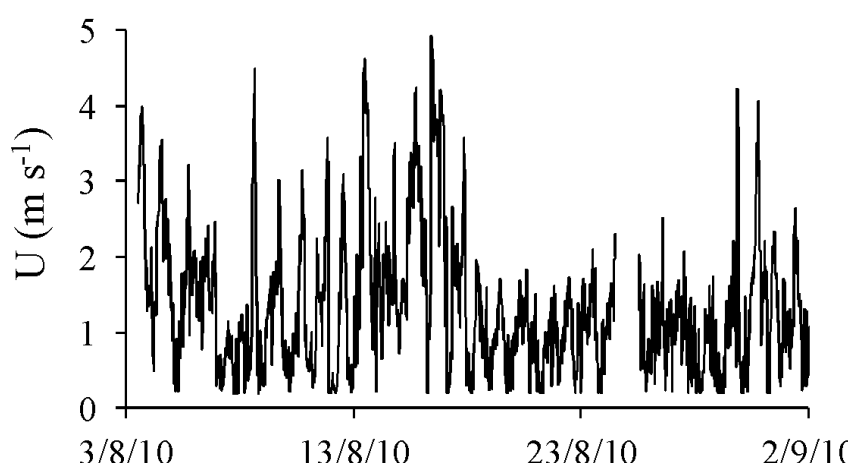

Figura 3. Intensidade do vento $U\left(\mathrm{~m} \mathrm{~s}^{-1}\right)$ medida na estação meteorológica da ESALQ/USP localizada a $20 \mathrm{~m}$ da área experimental

Na Figura 4 são apresentadas as medidas de duração do período de molhamento foliar feitas no tratamento IP e no tratamento ID. Como se observa nesta figura, os dados de DPM nos dias em que ocorreu a aplicação de irrigação foram excluídos da análise a fim de garantir que não haveria contaminação do sensor por água que não fosse proveniente da formação de orvalho, uma vez que não ocorreu precipitação no período. Nos dias em que há medidas em ambos os tratamentos observa-se que a DPM foi, em grande parte dos casos, maior no tratamento IP, como em 7 de agosto, quando a DPM no tratamento IP foi igual a $12,3 \mathrm{~h}$ e no tratamento ID, a $6,7 \mathrm{~h}$; na segunda metade do mês a DPM foi reduzida em ambos os tratamentos. Como apresentado na figura anterior, neste período o vento apresentou-se pouco intenso e estável, o que não contribuiu para a redução da DPM por secagem.

As relações entre as estimativas da DPM pelos modelos NHUR, DPO, CART e de P-M e as medidas feitas nos tratamentos de irrigação plena e deficitária, são apresentadas nas Figuras 5 e 6, respectivamente tal como, também, as simulações do modelo NHUR com o limiar de $90 \%$, do modelo DPO com

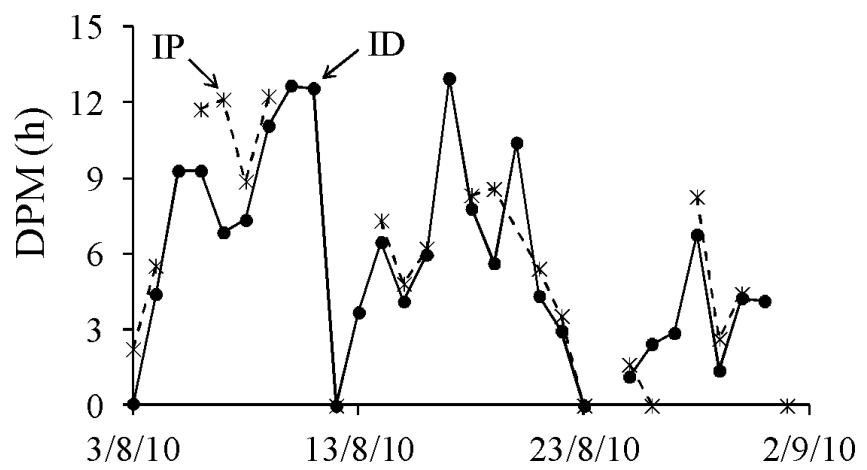

Figura 4. Duração do período de molhamento - DPM (h) medida no tratamento de irrigação deficitária (ID) e no tratamento de irrigação plena (IP) o limiar de $2{ }^{\circ} \mathrm{C}$ e do modelo de P-M com a temperatura e umidade do ar medidas na estação meteorológica. Seja para o tratamento ID (Figura 5) ou para o tratamento IP (Figura 6)

A.

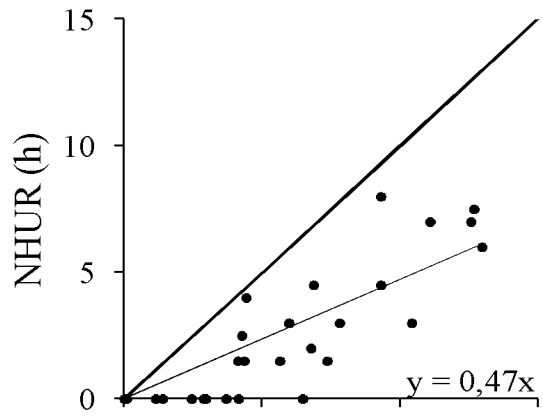

B.

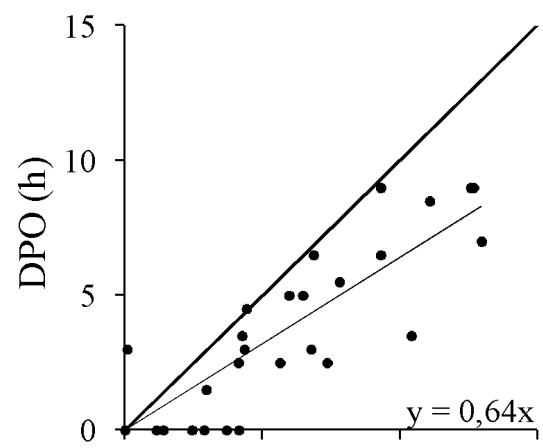

C.

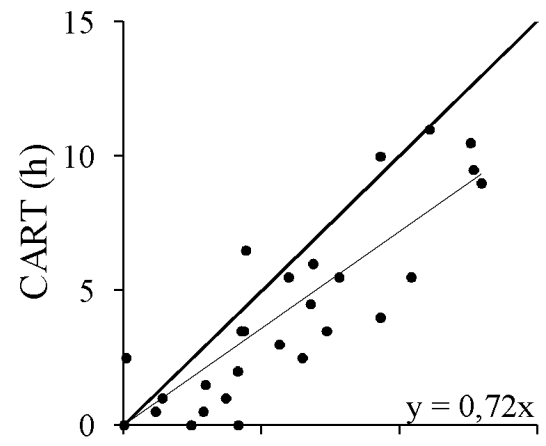

D.

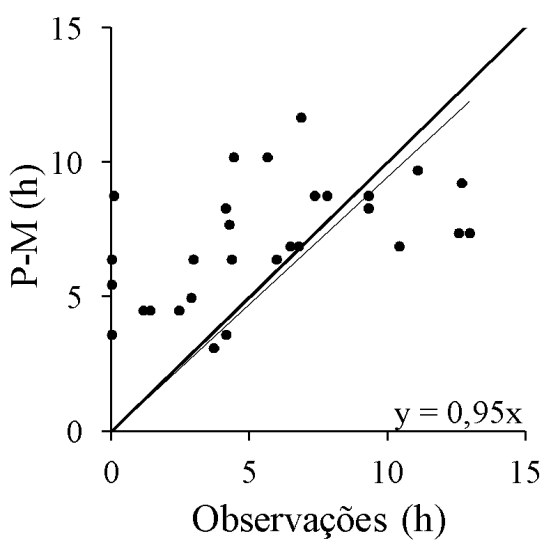

Figura 5. Duração do período de molhamento - DPM (h) estimada pelos modelos do número de horas com umidade relativa acima de determinado limiar (NHUR), empírico da depressão da temperatura do ponto de orvalho (DPO), de árvore de classificação e regressão (CART) e de Penmam-Monteith (P-M) e medida durante o experimento de campo no tratamento de irrigação deficitária (ID) 
A.

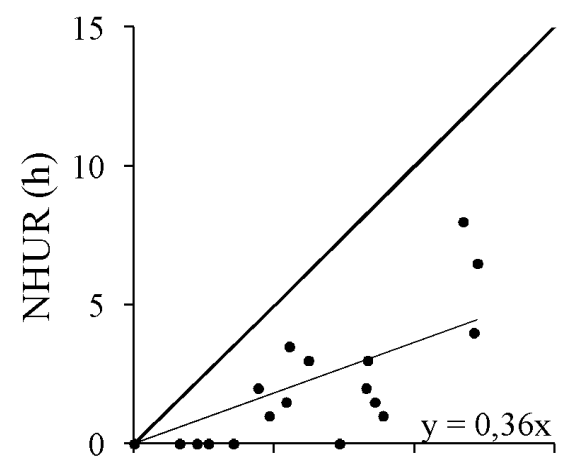

B.

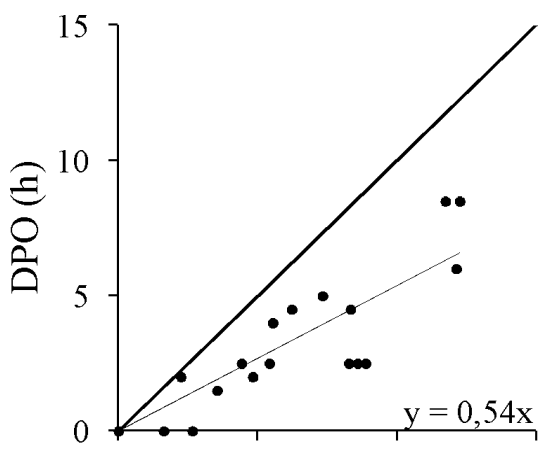

C.

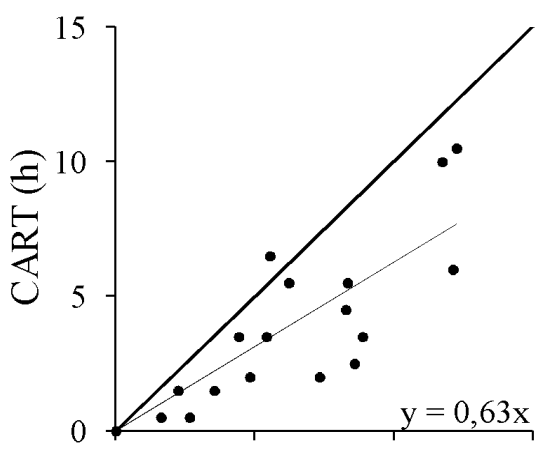

D.

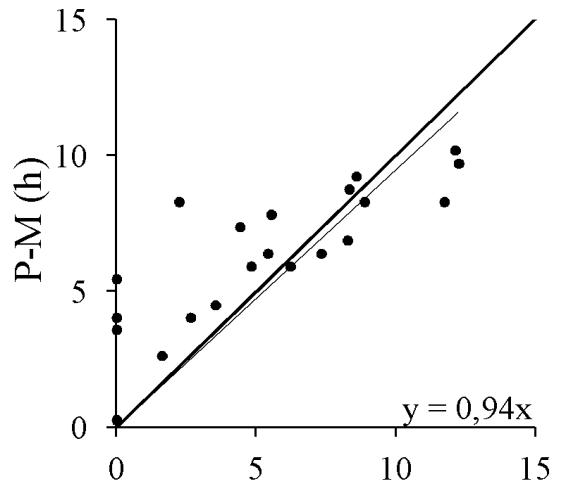

Observações $(\mathrm{h})$

Figura 6. Duração do período de molhamento - DPM (h) estimada pelos modelos do número de horas com umidade relativa acima de determinado limiar (NHUR), empírico da depressão da temperatura do ponto de orvalho (DPO), de árvore de classificação e regressão (CART) e de Penmam-Monteith (P-M) e medida durante o experimento de campo no tratamento de irrigação plena (IP)

todos os modelos subestimaram a duração do período de molhamento foliar. No tratamento ID as subestimativas foram de $53 \%$ com o modelo NHUR, $36 \%$ com o modelo DPO, $28 \%$ com o modelo CART e 5\% com o modelo de P-M; no tratamento IP, por sua vez, as subestimativas foram de $64 \%$ com o modelo NHUR, $46 \%$ com o modelo DPO, $37 \%$ com o modelo CART e $6 \%$ com o modelo de P-M. Um estudo feito por Lulu (2008) em vinhedo da cultivar 'Niagara Rosada' em Jundiaí, São Paulo, também resultou em subestimativas da DPM pelos modelos NHUR com o limiar de $90 \%$, DPO com o limiar de $2{ }^{\circ} \mathrm{C}$ e CART, enquanto que o modelo de P-M superestimou a DPM. Santos (2006) estimou a DPM em um gramado com os modelos DPO e CART para a estação seca em Piracicaba, São Paulo, e também observou subestimativas em relação às observações.

Os limiares empíricos de formação do orvalho dos modelos NHUR e DPO $\left(90 \%\right.$ e $2{ }^{\circ} \mathrm{C}$, respectivamente) foram calibrados para o clima local e para a cultura de feijão, conforme sugerido por Sentelhas (2004). Após a calibração os limiares que apresentaram os menores erros (Tabelas $1 \mathrm{e}$ 2) no modelo NHUR foram 84 e $83 \%$ para o tratamento ID e IP, respectivamente; para o modelo DPO os limiares com menores erros foram iguais a 2,6 e $2,7{ }^{\circ} \mathrm{C}$ para o tratamento

Tabela 1. Erro médio (EM), erro absoluto médio (EAM), erro máximo (EMax), raiz quadrada do erro quadrático médio (REQM $)$ e coeficiente de eficiência das previsões (E) entre a DPM medida e estimada pelos modelos do número de horas com umidade relativa acima de determinado limiar (NHUR), empírico da depressão da temperatura do ponto de orvalho (DPO), de árvore de classificação e regressão (CART) e de Penmam-Monteith (P-M) para o tratamento de irrigação deficitária (ID)

\begin{tabular}{lrrrrc}
\hline \multirow{1}{*}{ Modelo } & EM & EAM & EMax & REQM $_{p}$ & \multirow{2}{*}{ E } \\
\cline { 2 - 5 } & \multicolumn{3}{c}{ (h) } \\
NHUR $>84 \%$ & 0,73 & 1,70 & 8,92 & 2,61 & 0,56 \\
NHUR $>90 \%$ & 3,21 & 3,21 & 7,42 & 3,86 & 0,03 \\
DPO $<2{ }^{\circ} \mathrm{C}$ & 2,09 & 2,30 & 6,92 & 2,91 & 0,45 \\
DPO $<2,6^{\circ} \mathrm{C}$ & 0,75 & 2,60 & 8,42 & 2,55 & 0,58 \\
CART & 1,68 & 2,03 & 5,31 & 2,54 & 0,58 \\
P-M T $_{\text {ar }}$ e UR da estação & $-1,54$ & 3,04 & 8,68 & 3,72 & 0,10 \\
P-MT $_{\text {ar }}$ e UR do tratamento & $-0,26$ & 2,49 & 5,84 & 3,10 & 0,38 \\
\hline
\end{tabular}

Tabela 2. Erro médio (EM), erro absoluto médio (EAM), erro máximo (EMax), raiz quadrada do erro quadrático médio (REQM ) e coeficiente de eficiência das previsões (E) entre a DPM medida e estimada pelos modelos do número de horas com umidade relativa acima de determinado limiar (NHUR), empírico da depressão da temperatura do ponto de orvalho (DPO), de árvore de classificação e regressão (CART) e de Penmam-Monteith (P-M) para o tratamento de irrigação plena (IP)

\begin{tabular}{|c|c|c|c|c|c|}
\hline \multirow{2}{*}{ Modelo } & EM & EAM & EMax & REQM $_{p}$ & \multirow{2}{*}{$\mathbf{E}$} \\
\hline & \multicolumn{4}{|c|}{ (h) } & \\
\hline NHUR $>83 \%$ & 0,61 & 1,76 & 6,76 & 2,44 & 0,62 \\
\hline NHUR > $90 \%$ & 3,67 & 3,67 & 8,13 & 4,50 & $-0,29$ \\
\hline $\mathrm{DPO}<2{ }^{\circ} \mathrm{C}$ & 2,62 & 2,62 & 6,38 & 3,33 & 0,29 \\
\hline $\mathrm{DPO}<2,7^{\circ} \mathrm{C}$ & 0,84 & 1,74 & 6,26 & 2,41 & 0,63 \\
\hline CART & 2,12 & 2,21 & 6,13 & 2,97 & 0,44 \\
\hline P-M T $T_{a r}$ e UR da estação & $-0,96$ & 2,01 & 6,06 & 2,60 & 0,57 \\
\hline P-MT ${ }_{\mathrm{ar}}$ e UR do tratamento & 2,01 & 2,32 & 5,17 & 2,69 & 0,54 \\
\hline
\end{tabular}


ID e IP, respectivamente; no tratamento ID as subestimativas com os novos limiares foram reduzidas sendo iguais a $18 \%$ para ambos os modelos NHUR e DPO. No tratamento IP, por sua vez, as subestimativas foram de $19 \%$ com o modelo NHUR e $22 \%$ com o modelo DPO. As simulações com o modelo de P-M foram feitas de duas formas: com os dados de temperatura e umidade relativa do ar medidas na estação meteorológica da ESALQ/USP e com os dados dessas variáveis medidas no centro do tratamento sobre o dossel. A utilização dos dados obtidos sobre o dossel aumentou as subestimativas em ambos os tratamentos: o modelo subestimou as medidas de DPM em $16 \%$ no tratamento ID e em $37 \%$ no tratamento IP.

Os índices estatísticos calculados para as simulações são apresentados na Tabela 1 para o tratamento ID e na Tabela 2 para o tratamento IP, respectivamente. Para o tratamento ID (Tabela 1) o modelo NHUR melhorou seu desempenho com o limiar reduzido de $84 \%$ ao invés de $90 \%$, tradicionalmente utilizado. Os erros EM, EAM e REQM foram reduzidos com NHUR $>84 \%$ e somente o EMax aumentou em 1,5 h com esse novo limiar. A eficiência do modelo também aumentou significativamente com o coeficiente $\mathrm{E}$ indo de 0,03 para 0,56 . Kruit et al. (2008) calibraram o limiar do modelo NHUR para o gramado de uma estação meteorológica localizada em Haarweg, Holanda, na primavera, condições em que o limiar do modelo NHUR foi reduzido de $90 \%$ para $71 \%$. O modelo DPO teve seu desempenho aprimorado com o novo limiar de $2,6{ }^{\circ} \mathrm{C}$ ao invés de $2{ }^{\circ} \mathrm{C}$ sendo que os erros EM e REQM foram reduzidos e EAM e EMax aumentaram 0,3 e 1,4 h, respectivamente. A eficiência do modelo DPO aumentou 0,13 , indo de 0,45 para 0,58 . Os limiares empíricos do modelo CART não foram calibrados mas os erros das estimativas com esse modelo foram da mesma ordem de grandeza dos modelos NHUR e DPO com os limiares calibrados $\left(84 \%\right.$ e $\left.2,6^{\circ} \mathrm{C}\right)$. O coeficiente $\mathrm{E}$, por exemplo, foi igual ao do modelo DPO com o limiar de $2,6^{\circ} \mathrm{C}$ $(\mathrm{E}=0,58)$. De maneira geral e segundo Kim et al. (2010) o modelo CART apresenta uma acurácia na previsão da DPM foliar maior do que o modelo NHUR $>90 \%$ e que um modelo de redes neurais artificiais. Kim et al. (2002) comentam que a acurácia do modelo CART aumenta quando as medidas de intensidade do vento são corrigidas para a altura do sensor de molhamento. O desempenho do modelo de P-M melhorou para as simulações feitas com os dados coletados sobre dossel; todos os erros foram reduzidos e a eficiência do modelo em simular os dados observados aumentou de 0,10 para 0,38 .

Todos os erros das simulações feitas pelo modelo NHUR para os dados do tratamento IP (Tabela 2) foram reduzidos quando o limiar de $83 \%$ foi utilizado ao invés de $90 \%$. A eficiência do modelo melhorou significativamente com o novo limiar indo de -0,29, valor negativo indicando que a média das observações representava melhor os dados observados do que as próprias simulações, para 0,62. Da mesma forma que para o modelo NHUR, os erros das simulações com o modelo DPO foram reduzidos quando o limiar de $2,7^{\circ} \mathrm{C}$ foi adotado ao invés de $2{ }^{\circ} \mathrm{C}$ e a eficiência do modelo, embora positiva $(0,29)$ aumentou para 0,63 com o novo limiar. Nenhuma modificação foi feita nos limiares empíricos do modelo CART mas como para o tratamento ID os erros são comparáveis aos erros dos modelos NHUR e DP com os limiares de $83 \%$ e 2,7 ${ }^{\circ} \mathrm{C}$ e o coeficiente $\mathrm{E}$ das simulações foi positivo $(\mathrm{E}=0,44)$ demonstrando que o modelo foi eficiente em simular os dados de DPM observados. Apesar de ser um modelo empírico o modelo CART utiliza limiares empíricos de três variáveis (DPO, umidade relativa do ar e intensidade do vento) explicação provável para que a duração do período de molhamento foliar estimada por este modelo apresente melhores resultados do que os modelos empíricos que utilizam o limiar de uma única variável. Diferente das simulações feitas para o tratamento ID, as simulações feitas com o modelo de P-M, tanto com os dados da estação meteorológica da ESALQ/USP quanto com os dados observados sobre o dossel, não apresentaram diferenças significativas. Em ambos os casos os erros foram próximos e a eficiência do modelo diminuiu levemente de 0,57 para 0,54 , quando os dados obtidos sobre o dossel foram utilizados como entrada do modelo.

A raiz quadrada do erro quadrático médio das observações de DPM (REQM ) foi igual a 3,92 h para o tratamento ID e 3,96 $\mathrm{h}$ para o tratamento IP. De acordo com as Tabelas 1 e 2, apenas as simulações feitas com o modelo NHUR $>90 \%$ apresentaram um erro $\left(\mathrm{REQM}_{\mathrm{p}}\right)$ superior ao erro das próprias observações de DPM, em ambos os tratamentos $(3,86 \mathrm{~h}$ para o tratamento ID e 4,50 h para o tratamento IP).

\section{Conclusões}

1. O efeito microclimático da diferença da transpiração das plantas irrigadas e das plantas sob déficit hídrico resultou, em grande parte dos casos, em maior duração do período de molhamento foliar no tratamento com irrigação plena.

2. A calibração dos limiares dos parâmetros empíricos dos modelos NHUR e DPO para o clima local e para a cultura do feijão reduziu o erro entre as simulações e as observações da duração do período de molhamento foliar.

3. Os erros das simulações feitas com o modelo CART com limiares não calibrados localmente foram da mesma ordem de grandeza dos obtidos pelos demais modelos.

4. Os erros das simulações com o modelo físico de P-M feitas com os dados medidos numa estação meteorológica foram pequenos quando o dossel apresentou condições microclimáticas similares às reinantes na estação meteorológica.

5. Para um dossel sob condições hídricas limitantes com o microclima modificado, o modelo de P-M teve seu desempenho melhorado pela utilização de dados obtidos sobre o dossel.

\section{Agradecimentos}

Os autores agradecem o apoio financeiro do Projeto de Cooperação Internacional à CAPES-Wageningen Univeristy and Research Centre (Processo 019/06) e da Fundação de Apoio à Pesquisa do Estado de São Paulo - FAPESP (Processo 2009/02117-7).

\section{Literatura Citada}

Allen, R. G.; Pereira, L. S.; Raes, D.; Smith, M. Crop evapotranspiration - Guidelines for computing crop water requirements - Rome: FAO, 1998. 300p. FAO Irrigation and Drainage Paper 56. 
Bregaglio, S.; Donatelli, M.; Confalonieri, R.; Acutis, M.; Orlandini, S. Multi metric evaluation of leaf wetness models for large-area application of plant disease models. Agricultural and Forest Meteorology, v.151, p.1163-1172, 2011.

Gillespie, T. J.; Srivastava, B.; Pitblado, R.E. Using operational weather data to schedule fungicide sprays on tomatoes in southern Ontario, Canada. Journal of Applied Meteorology, v.32, p.567-573, 1993.

Gleason M. L.; Taylor, S. E.; Loughin, T. M.; Koehler, K. J. Development and validation of an empirical model to estimate the duration of dew periods. Plant Disease, v.78, p.1011-1016, 1994.

Hanba, Y. T.; Moriya, A.; Kimura, K. Effect of leaf surface wetness and wettability on photosynthesis in bean and pea. Plant, Cell and Environment, v.27, p.413-421, 2004.

Huber, L.; Itier, B. Leaf wetness duration in a field bean canopy. Agricultural and Forest Meteorology, v.51, p.281-292, 1990.

Ishibashi, M.; Terashima, I. Effects of continuous leaf wetness on photosynthesis: adverse aspects of rainfall. Plant, Cell and Environment, v.18, p.431-438, 1995.

Kim, K. S.; Gleason, M. L.; Taylor, S. E. Forecasting sitespecific leaf wetness duration for input to disease-warning systems. Plant Disease, v.90, p.650-656, 2006.

Kim, K. S.; Taylor, S. E.; Gleason, M. L.; Koehler, K. J. Model to enhance site-specific estimation of leaf wetness duration. Plant Disease, v.86, p.179-185, 2002.

Kim, K. S.; Taylor, S. E.; Gleason, M. L.; Villalobos, R.; Arauz, L. F. Estimation of leaf wetness duration using empirical models in northwestern Costa Rica. Agricultural and Forest Meteorology, v.129, p.53-67, 2005.

Kim, K. S.; Taylor, S.; Gleason, M. L.; Nutter Jr., F. W.; Coop, L. B.; Pfender, W. F.; Seem, R. C.; Sentelhas, P. C.; Gillespie, T. J.; Dalla Marta, A.; Orlandini, S. Spatial portability of numerical models of leaf wetness duration based on empirical approaches. Agricultural and Forest Meteorology, v.150, p.871-880, 2010.

Kruit, R. J. W.; Jacobs, A. F. G.; Holtslag, A. A. M. Measurements and estimates of leaf wetness over agricultural grassland for dry deposition modeling of trace gases. Atmospheric Environment, v.42, p.5304-5316, 2008.

Letts, M. G.; Mulligan, M. The impact of light quality and leaf wetness on photosynthesis in north-west Andean tropical montane cloud forest. Journal of Tropical Ecology, v.21, p.549-557, 2005.
Lulu, J. Duração do período de molhamento em vinhedo de 'Niágara Rosada' e sua relação com a ocorrência de míldio (Plasmoparaviticola). Piracicaba: ESALQ/USP, 2008. 188p. Tese Doutorado

Magarey, R. D.; Russo, J. M.; Seem, R. C.; Gadoury, D. M.Surface wetness duration under controlled environmental conditions. Agriculturaland Forest Meteorology, v.128, p.111-122, 2005.

Marcuzzo, L. L.; Fernandes, J. M. C.; Becker, W. F. Influência da temperatura e da duração do molhamento foliar na severidade da mancha bacteriana do tomateiro. Summa Phytopathologica, v.35, p.229-230, 2009.

Monteith, J. L.; Unsworth, M. H. Principles of environmental physics. 2.ed. New York: Edward Arnold, 1990. 291p.

Nash, J. E.; Sutcliffe, J. V. River flow forecasting through conceptual models I. A discussion of principles. Journal of Hydrology, v.10, p.282-290, 1970.

Papastamati, K.; McCartney, H. A.; Bosch, F. van den. Modelling leaf wetness duration during the rosette stage of oilseed rape.Agricultural and Forest Meteorology, v.123, p.69-78, 2004.

Santos, E. A. Duração do período de molhamento foliar: medida com sensores eletrônicos, variabilidade espacial em culturas e estimativa com modelos empíricos. Piracicaba: ESALQ/ USP, 2006. 56p. Dissertação Mestrado

Sentelhas, P. C. Duração do período de molhamento foliar: Aspectos operacionais da sua medida, variabilidade espacial em diferentes culturas e em sua estimativa a partir do modelo de Penman-Monteith. Piracicaba: ESALQ/USP, 2004. 182p. Tese Livre-Docência

Sentelhas, P. C.; Gillespie, T. J. Estimating hourly net radiation for leaf wetness duration using the Penman-Monteith equation.Theoretical and Applied Climatology, v. 91, p.205-215, 2008.

Sentelhas, P. C.; Gillespie, T. J.; Gleason, M. L.; Monteiro, J. E. B. A.; Pezzopane, J. R. M.; Pedro Jr., M. J. Evaluation of a Penman-Monteith approach to provide "reference" and crop canopy leaf wetness duration estimates. Agricultural and Forest Meteorology, v.141, p.105-117, 2006.

Weiss, A.; Lukens, D. L.; Norman, J. M.; Steadman, J. R. Leaf wetness in dry beans under semi-arid conditions. Agricultural and Forest Meteorology, v.48, p.149-162, 1989. 